\title{
GEOMETRICAL NONLINEAR ANALYSIS OF CURVED I-GIRDERS UNDER BENDING USING MIXED FINITE ELEMENT METHOD
}

\author{
By Yoshitaka SUETAKE*, Masaharu HIRASHIMA** and Teruhiko YODA**
}

\begin{abstract}
The purpose of the present paper is to examine the influence of flanges on the stength of curved I-girders under bending, in which the strength of curved I-girders with flanges is investigated using mixed finite element approach. Lagrange's multipliers method is used for the summation of element matrices along junctions of a web and flanges, and Newton-Raphson method and incremental procedure are employed for nonlinear anaysis. It appears from numerical results that interaction between a web and a compression flange can not be ignored for determination of the strength of curved I-girders.
\end{abstract}

Keywords: nonlinear, curred i-girders, F.E.M.

\section{INTRODUCTION}

Curved I-girders are being frequently used as structural members in the design of highway bridges. However, there exist only a few design criteria for curved I-girders. Some of them are incorporated into the BS $5400^{1)}$, AASHTO ${ }^{2)}$, DASt Ri $012^{3)}$, as a recomendation. Similar circumstances will be observed in our country.

A number of investigations concerning curved girders have been presented focusing on a web panel of those girders. Several excellent papers in these areas have been contributed by Culver, C. G. and Dym, C. L. ${ }^{4) ~ 6)}$, Dabrowski, R. and Wachowiak, J. ${ }^{7)}$, Abdel-Sayed ${ }^{8)}$ who analyzed the web panel by applying a linearized cylindrical thin shell theory. Mikami, I., Furunishi, K. and Yonezawa, H. ${ }^{9}$, employed nonlinear thin shell theory for the problem. Kuranishi, S. and Hiwatashi, S. ${ }^{10}$. used the finite element method for the analysis of the panel, in which strain-controled edge conditions are adopted under the existance of vertical stiffeners. An experimental study about bending strength of a web panel was made by Nakai, H., Kitada, T. and Ohminami, R. ${ }^{11}$ yielding valuable data. These investigations are mainly concerned with behavior of an isolated web panel. However, it has been pointed out that the effects of flange stiffness on the strength of curved I-girders cannot be ignored, so that the overall analysis of curved I-girders including flanges should be made. Hiwatashi, S. and Kuranishi, S. ${ }^{12)}$ reported general elastic behavior of curved I-girders, using the finite element method under the strain-controled edge condition, in which the total model with flanges is compared with an isolated web model, and effective width of web plates, resisting bending moment of girder, vertical buckling of flange plates are considered. Unfortunately, the behavior of flanges is not fully considered quantitatively. Fujii, K. and Ohmura, H. ${ }^{13)}$

* Student Member of the Japan Society of Civil Engineers. Engineering Research Laboratory, Waseda University, Tokyo.

** Member of the Japan Society of Civil Engineers. Department of Civil Engineering, Waseda University, Tokyo. 
investigated overall behavior of curved I-girders through F. E. M. analysis, in which data about flange rotations are included. They apply loads so as to statisfy the compatibility condition of displacements at both ends, introducing displacement field of beam theory. The influence of flanges and curvature on the behavior of a web panel was examined and torsional buckling of a compression flange is considered. In order to develop a design criteria, these investigations must be extended to the overall analysis of curved I-girders.

In this paper, nonlinear analysis of curved I-girders between vertical stiffeners under bending is dealt with, in which mixed finite element approach, which makes the formulation of element matrices easy and the accuracy of which is relatively good, is employed. In the procedure presented, element matrices along junctions are summed up with the help of Lagrange's multipliers method to form fully compatible finite elements ${ }^{14)}$. The distributions of stress at both ends are confined to be linear for a web and uniform for flanges. The loading edges of a web are assumed to be simply supported, and moments along the loading edges of flanges are assumed to be zero. Geometrical nonlinearity of total curved I-girders is investigated by changing flange width-thickness ratio, radius of curvature and web depth-thickness ratio. As a result, it turned out that the influence of flanges on the behavior of web can not be ignored for the determination of strength of curved I-girders, and that web depqh-thickness ratio affects web deflection and geometrical nonlinearity increases with the curvature of the girders.

\section{FINITE DISPLACEMENT FORMULATION FOR I-GIRDERS}

In this paper, a nonlinear incremental element equation is deduced by using nonlinear straindisplacement relation, and an incremental approach together with Newton-Raphson method is employed for finite displacement analysis, in which Lagrange's multiplier method is used in the summation of element matrices along junctions of a web and flanges. The principal differences in formulation from Ref. 15) are the introcuction of nonlinear strain-displacement relation throughout the formulation and the application of iterative procedure to the nonlinear analysis.

(1) Derivation of nonlinear element equation

With a view to Ref. 15), we obtain the following nonlinear incremental element equation to a triangular membrane and bending element;

$$
\left[\begin{array}{cc}
A_{\text {inc. }}+C^{\prime} & b^{T} H^{T} \\
H b & -G
\end{array}\right]\left\{\begin{array}{c}
\Delta r \\
\Delta M
\end{array}\right\}=\left\{\begin{array}{c}
\Delta R \\
-\Delta \phi
\end{array}\right\}
$$

$; \boldsymbol{A}_{\text {inc. }}=\boldsymbol{A}+2\left(\boldsymbol{A}_{1}+\boldsymbol{A}_{1}^{T}+\boldsymbol{A}_{2}\right), C^{\prime}=C_{L}^{\prime}+C_{N L}^{\prime}, \quad W=b r$

where $\boldsymbol{A}$ : linearized in-plane stiffness matrix, $C_{L}^{\prime}$ : linearized initial stress matrix, $G$ : flexibility matrix for bending, $\boldsymbol{r}:$ nodal displacement vector, $\boldsymbol{M}:$ element boundary moment vector, $\boldsymbol{R}:$ nodal force vector, $\phi$ : element boundary angle vector, $\boldsymbol{W}$ : out-of-plane displacement vector, matrix $\boldsymbol{H}$ represents equilibrium between out-of-plane nodal forces and element boundary moments ${ }^{15)}$, and matrices $\boldsymbol{A}_{1}, \boldsymbol{A}_{2}$ and $C_{N L}^{\prime}$ which are not considered in Ref. 15) are originate from nonlinearity of strain-displacement relation :

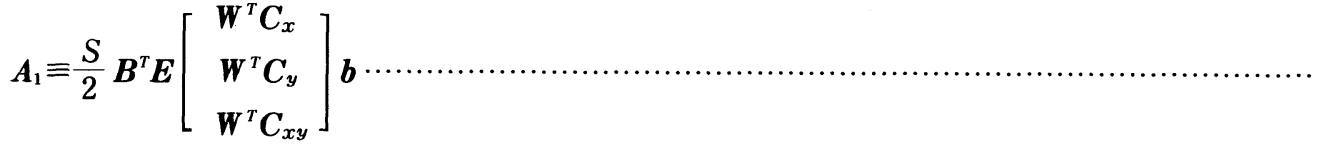

$$
\begin{aligned}
& \boldsymbol{A}_{2} \equiv \frac{S}{2} \boldsymbol{b}^{T}\left[\begin{array}{lll}
\boldsymbol{C}_{x}^{T} \boldsymbol{W} & \boldsymbol{C}_{y}^{T} \boldsymbol{W} & \boldsymbol{C}_{x y}^{T} \boldsymbol{W}
\end{array}\right] \boldsymbol{E}\left[\begin{array}{c}
\boldsymbol{W}^{T} \boldsymbol{C}_{x} \\
\boldsymbol{W}^{T} \boldsymbol{C}_{y} \\
\boldsymbol{W}^{T} \boldsymbol{C}_{x y}
\end{array}\right] \boldsymbol{b} \\
& \boldsymbol{C}_{N L}^{\prime} \equiv \frac{S}{2} \boldsymbol{b}^{T}\left[T_{N L x} C_{x}+T_{N L y} C_{y}+T_{N L x y} C_{x y}\right] \boldsymbol{b} ; \boldsymbol{E}\left[\begin{array}{c}
\boldsymbol{W}^{T} \boldsymbol{C}_{x} \boldsymbol{W} \\
\boldsymbol{W}^{T} \boldsymbol{C}_{y} \boldsymbol{W} \\
\boldsymbol{W}^{T} C_{x y} \boldsymbol{W}
\end{array}\right] \equiv\left\{\begin{array}{c}
T_{N L x} \\
T_{N L y} \\
T_{N L x y}
\end{array}\right)
\end{aligned}
$$


in which $S$ is area of the element, $B$ is a matrix representing linearized strain-displacement relation, $C_{x}$, $C_{y}$ and $C_{x y}$ are matrices that are obtained through multiplication of the interpolation functions, and $\boldsymbol{E}$ is an elastic matrix for plane stress problems ${ }^{15)}$.

In each incremental step, nonlinear analysis is performed by the use of modified Newton-Raphson method with the help of the following equation :

$$
\left[\begin{array}{cc}
\boldsymbol{A}^{\prime} & \boldsymbol{b}^{T} \boldsymbol{H}^{T} \\
\boldsymbol{H} \boldsymbol{b} & -\boldsymbol{G}
\end{array}\right]\left\{\begin{array}{c}
\boldsymbol{r} \\
\boldsymbol{M}
\end{array}\right\}=\left\{\begin{array}{c}
\boldsymbol{R} \\
-\boldsymbol{\phi}
\end{array}\right\} ; \boldsymbol{A}^{\prime} \equiv \boldsymbol{A}_{1}+2 \boldsymbol{A}_{1}^{T}+\boldsymbol{A}_{2}
$$

in which the iteration for the convergence is executed until the unbalanced forces become $1.0 \%$ of the incremental loads.

(2) Summation of element matrices along junctions

If more than two elements meet at a common intersection, attention should be paid for the summation of element matrices along the line ${ }^{14)}$ (for example, along junctions of a web and flanges of I-girders). The mixed finite element approach does not allow us to superpose element matrices along the junctions, since moments are not continuous along the junctions, while rotations are continuous on the junctions. As shown in Fig. 1, we introduce boundary moments $M_{I}, M_{J}$ and $M_{K}$ along the junctions as independent unknown papameters. Supplementing the lack of boundary conditions, we consider the equilibrium condition for those moments. The equilibrum condition requires that

$$
M_{I}+M_{J}+M_{K}-M_{\mathrm{ex}}=0
$$

where $M_{\mathrm{ex}}$ is an external moment acting on the junctions (zero in this paper). In order to introduce Eq. (6) into the total structural system, the following generalization should be made

$$
\begin{aligned}
& \cdots I \cdots \cdots J \cdots \cdots, \cdots \\
& {\left[\begin{array}{lllllll}
0 & 1 & \mathbf{0} & 1 & \mathbf{0} & 1 & 0
\end{array}\right] \boldsymbol{M}_{\mathrm{tot} .}-M_{\mathrm{ex} .}=0}
\end{aligned}
$$

in which $\boldsymbol{M}_{\text {tot. }}$ is the total unknown moment vector whose size is $n \times 1$. If all the junctions are divided into $\mathrm{m}$ parts, $\mathrm{m}$ equations like Eq. (7) are obtained. It follows from this that

$$
k_{(m \times n)}^{\prime} \boldsymbol{M}_{\text {tot. }}-\underset{(m \times 1)}{M_{\text {ex. }}}=\mathbf{0}
$$

For later use, we rewrite Eq. (8) as

$$
\left[\begin{array}{ll}
0 & \boldsymbol{k}^{\prime}
\end{array}\right]\left\{\begin{array}{c}
\boldsymbol{r}_{\text {tot. }} \\
\boldsymbol{M}_{\text {tot. }}
\end{array}\right\}-\boldsymbol{M}_{\mathrm{ex} .}=\boldsymbol{k} \boldsymbol{u}-\boldsymbol{M}_{\mathrm{ex} .}=\mathbf{0} ; \boldsymbol{k}=\left[\begin{array}{ll}
\mathbf{0} & \boldsymbol{k}^{\prime}
\end{array}\right], \boldsymbol{u}=\left\{\begin{array}{c}
\boldsymbol{r}_{\text {tot. }} \\
\boldsymbol{M}_{\text {tot. }}
\end{array}\right\}
$$

in which $\boldsymbol{r}_{\text {tot. }}$ is a total unknown nodal displacements vector. Assembling element equation (5) without considering the compatibility conditions along the junctions yields

$$
\bar{K} \boldsymbol{u}=\boldsymbol{f}
$$

where

$\bar{K}$ : global matrix for the incompatible stuctural system,

$f$ : load-rotation angle vector.

Equation (10) is unsolvable, because the coefficient matrix $\overline{\boldsymbol{K}}$ in Eq. (10) is singular for want of boundary conditions. We should supplement the lack of boundary conditions by using Eq. (9) with the help of Lagrange's multipliers method. Since the matrix $\bar{K}$ is symmetric, a functional $\Pi_{R}$ which yields Eq. (10) is given by

$$
\Pi_{R}=\frac{1}{2} \boldsymbol{u}^{T} \bar{K} \boldsymbol{u}-\boldsymbol{u}^{T} \boldsymbol{f}
$$

Therefore, the modified functional $\Pi_{R}^{*}$ is derived from Eqs. (9) and (11) as follows:

$$
\Pi_{R}^{*}=\Pi_{R}+\lambda^{T}\left(\boldsymbol{k} \boldsymbol{u}-\boldsymbol{M}_{\mathrm{ex}}\right)=\frac{1}{2} \boldsymbol{u}^{T} \overline{\boldsymbol{K}} \boldsymbol{u}-\boldsymbol{u}^{T} \boldsymbol{f}+\lambda^{T}\left(\boldsymbol{k} \boldsymbol{u}-\boldsymbol{M}_{\mathrm{ex}}\right)
$$

where $\lambda$ is Lagrange's multipliers vector, the stationary condition of $\Pi_{R}^{*}$ yields 
$\left[\begin{array}{cc}\bar{K} & k^{T} \\ k & 0\end{array}\right]\left\{\begin{array}{c}u \\ \lambda\end{array}\right\} \equiv\left\{\begin{array}{c}f \\ M_{\mathrm{ex} .}\end{array}\right\}$

Note that $\boldsymbol{M}_{\text {ex. }}$ is a zero vector in this paper.

\section{MODEL OF CURVED I-GIRDER}

General view of a curved I-girder is shown in Fig. 2. The numerical models employed in this paper are classified into five types with respect to curvature parameter $Z: Z=a^{2} \sqrt{1-\nu^{2}} / R t_{w}$ in which $a$ is distance between vertical stiffeners, web depth-thickness ratio $h / t_{w}$, and aspect ratio $a / h$, which are tabulated in Table $1^{16)}$. Flange width-thickness ratios $b / t_{f}$ used in the models are 8.57, 12.0 and 20.0. Mechanical properties of steel are the same in all Types of models, that is, Young's modulus $: E=2.1 \times$ $10^{6}\left[\mathrm{~kg} / \mathrm{cm}^{2}\right]$ i. e. $2.06 \times 10^{5}[\mathrm{MPa}]$, Poisson's ratio: $\nu=0.3$. Yielding stress $\sigma_{Y}$ is assumed to be 2400 [kg/ $\left.\mathrm{cm}^{2}\right]$ i. e. $235[\mathrm{MPa}]$ for Types $1,4,5$ and $3600\left[\mathrm{~kg} / \mathrm{cm}^{2}\right]$ i. e. $353[\mathrm{MPa}]$ for Types $2,3$. Out-of-plane displacements and moments with respect to a generating line are assumed to be zero on both ends of a web panel, and plate bending moments at the ends of flanges are also assumed to be zero. In this paper, owing to the presence of vertical stiffeners at both ends, it is assumed that distance between an upper and a lower flanges remains constant at the ends, and that out-of-plane displacements at Points $\mathrm{C}$ and $\mathrm{C}^{\prime}$ are taken to be zero, while finite element representation for vertical stiffeners is not performed. It will be expected that the variation of normal stress across the flange width due to curvature is assumed negligible, since curvatures of the present models are small ${ }^{4) .17)}$. Distributions of stress at both ends are confined to be linear for a web and uniform for flanges in order to simplify the problem. Therefore, the compatibility of displacements at both ends of the models cannot necessarily be satisfied between analytical models and real curved I-girders. Numerical analysis is performed right half of the models due to symmetry of the models. A web panel of this half model is divided into 100 elements and its upper or lower flange plate is divided into 40 elements respectively.

Table 1 Classification of Models.

\begin{tabular}{c|c|c|c}
\hline Type & $\begin{array}{c}\text { Curvature } \\
\text { parameter } \\
\text { Z }\end{array}$ & $\begin{array}{c}\text { web depth- } \\
\text { thickness ratio } \\
\text { h/tw }\end{array}$ & $\begin{array}{c}\text { Aspect } \\
\text { ratio } \\
\mathrm{a} / \mathrm{h}\end{array}$ \\
\hline 1 & 7.15 & 150 & 1.0 \\
2 & 3.18 & 100 & 1.0 \\
3 & 7.15 & 100 & 1.5 \\
4 & 4.29 & 150 & 1.0 \\
5 & 2.15 & 150 & 1.0 \\
\hline
\end{tabular}

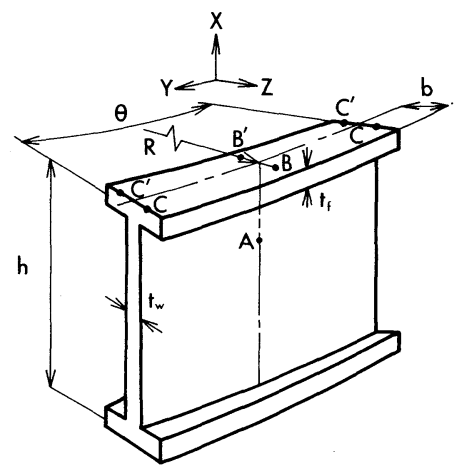

Fig. 2 General view of a curved I-girder.

\section{NUMERICAL RESULTS AND DISCUSSION}

Value of each parameter adopted in this paper is not always ordinary one. Particularly, wide flanges are selected in order to observe buckling behavior of a compression flange.

(1) Torsional buckling of compression flange

A compression flange of curved I-girders deforms as shown in Figs. 3 and 4 in the case of Type 4. As will be observed in Fig. 3(b), torsional deformation of a flange is unambiguous. Figures. $5 \sim 7$ also show torsional deformation of compression flanges which equals rotation of $\mathrm{B}^{-\mathrm{B}^{\prime}}$ in Fig. 2. The results of Types 3 and 4 (Table 1) as typical examples are shown in Figs. 5 and 6, in which nondimensional flange stress $\sigma_{f} / \sigma_{Y}$ is plotted against to the torsional angle of flange plate. Figure. 7 shows the results of Types 1 


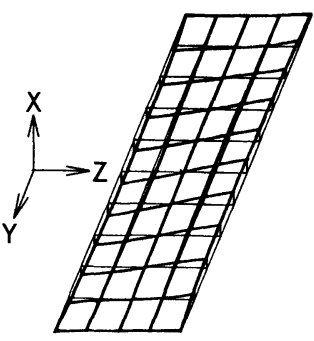

(a) nondimensional flange stress $\sigma_{f} / \sigma_{Y}=0.5$

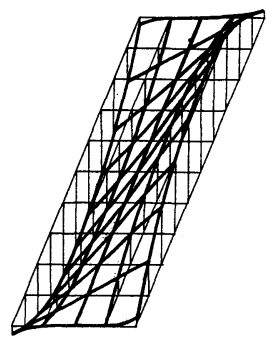

(b) nondimensional flange stess $\sigma_{f} / \sigma_{Y}=1.0$

Fig. 3 Overall deformation of a compression flange (Type $4, b / t_{f}=20.0$ ).

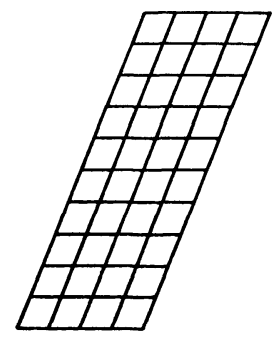

(a) nondimensional flange stress $\sigma_{f} / \sigma_{Y}=0.5$

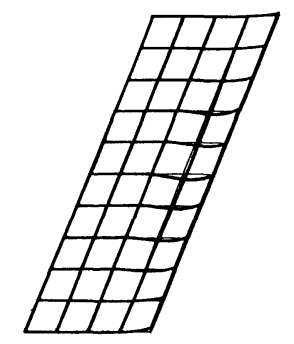

(b) nondimensional flnge stress $\sigma_{f} / \sigma_{Y}=1.0$

Fig. 4 Overall deformation of a compression flange (Type $4, b / t_{f}=8.57$ ).

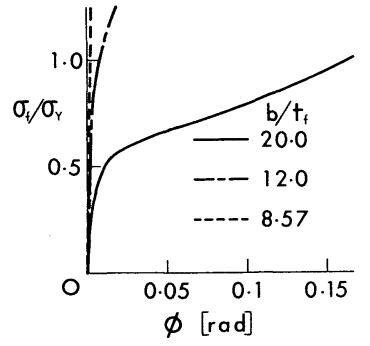

Fig. 5 Rotation of a compression flange (Type 3 ).

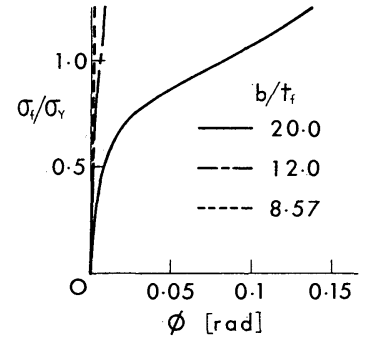

Fig. 6 Rotation of a compression flange (Type 4).

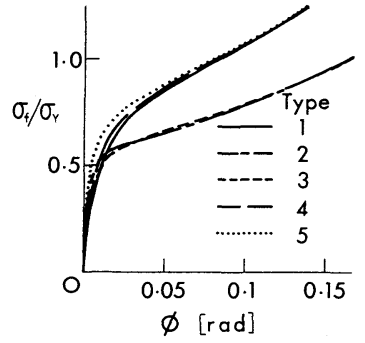

Fig. 7 Flange rotation for each type $\left(b / t_{f}=20.0\right)$.

to 5 , in which $b / t_{f}=20.0$. It is obvious from these figures that torsional buckling of a compression flange occurs in the case of $b / t_{f}=20.0$. This torsional buckling behavior may be confirmed from Fig. 3(a) and (b). On the other hand, in the case of $b / t_{f}=8.57$ and 12.0, compression flanges scarcely deform. Similar tendency is observed for the other Types. It can be seen from Fig. 7 that each buckling load of a compression flange is $\sigma_{f} / \sigma_{Y}=1.0$ for Types $1,4,5$, and $\sigma_{f} / \sigma_{Y}=0.7$ for Types 2,3 . If we consider an outstanding compression flange as a rectangular plate of which one edge is free and the other three edges are simply supported, we can calculate theoretical values of the buckling load, that is, $\sigma_{f} / \sigma_{Y}=1.0$ for Types 1, 4, 5, $\sigma_{f} / \sigma_{Y}=0.71$ for Type 2 and $\sigma_{f} / \sigma_{Y}=0.67$ for Type $3^{18)}$. Comparison between the analytical results and the above theoretical values suggests that curvature scarcely affects the buckling load of a compression flange under the present boundary conditions when $Z \leq 7.15$. From Fig. 7, it is pointed out that the flange buckling loads for Types 2 and 3 are equal. Curvature parameters $Z$ and aspect ratios of these two types different from each other, while their web depth-thickness ratios are the same $h / t_{w}=100$. It follows from this that aspect ratio scarcely affects the flange buckling load when $Z \leq 7.15, a / h \geq 1.0$, since it is expected from the consideration on the results for Types 1,4 and 5 that curvature does not play a key role on the buckling behavior of a compression flange.

\section{(2) Deflection of a web}

Web deflection of curved I-girders are shown in Figs. 8 12. Figures. 8 and 9 show overall web deflection for $b / t_{f}=20.0$ and $b / t_{f}=8.57$ respectively. As is evident from these figures, web deflection is influenced by the presence of torsional buckling of a compression flange. In the case of $b / t_{f}=20.0$, in which flange buckling occurs, deflection mode of compression region of a web remains a half wave of sinusoidal curve (first mode) whether load is large or not (Fig. 8(a) and (b)). On the other hand, in the case of $b / t_{f}=8.57$, one and a half waves (third mode) are observed (Fig. 9(a) and (b)). Even if load level is low, the third mode appears near the junction between a compression flange and a web (Fig. 9(a)), and it extends all over the compression region as load increases (Fig. 9(b)). It appears from these results that 


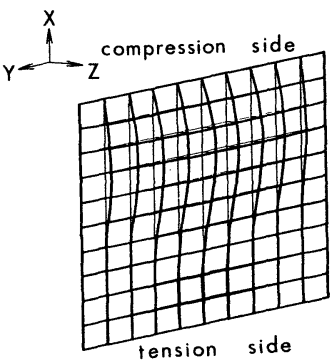

(a) $\sigma_{f} / \sigma_{Y}=0.5$

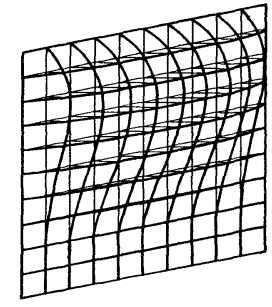

(b) $\sigma_{f} / \sigma_{Y}=1.0$

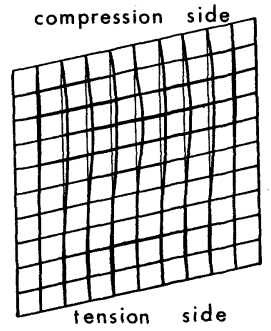

(a) $\sigma_{f} / \sigma_{Y}=0.5$

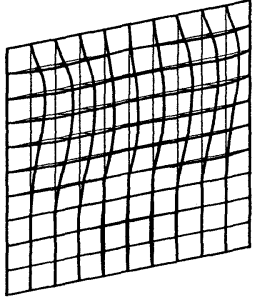

(b) $\sigma_{f} / \sigma_{Y}=1.0$

Fig. 8 Overall deformation of a web (Type $4, b / t_{f}=20.0$ ).

Fig. 9 Overall deformation of a web (Type $4, b / t_{f}=8.57$ ).

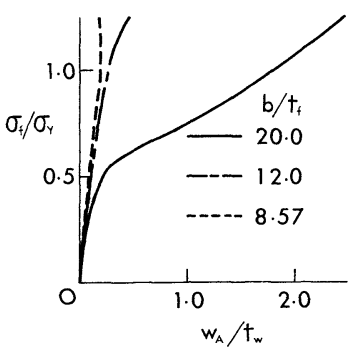

Fig. 10 Web deflection at Point A (Type 3).

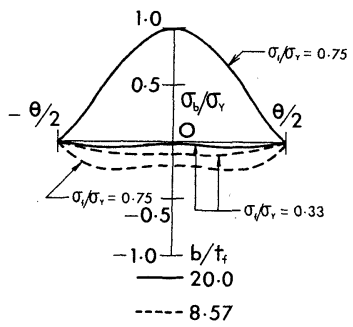

Fig. 13 Nondimensional bending stress $\sigma_{b} / \sigma_{Y}$ along the junction (Type 3).

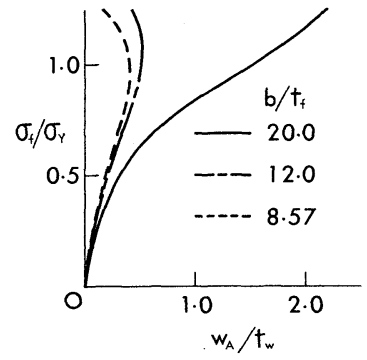

Fig. 11 Web deflection at Point A (Type 4)

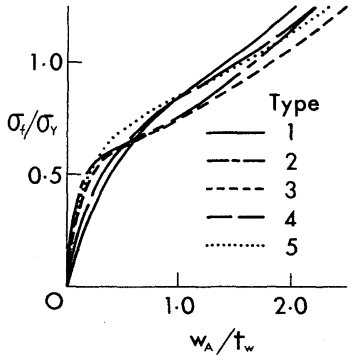

Fig. 12 Web deflection at Point $\mathrm{A}$ for each type $\left(b / t_{f}=20.0\right)$.

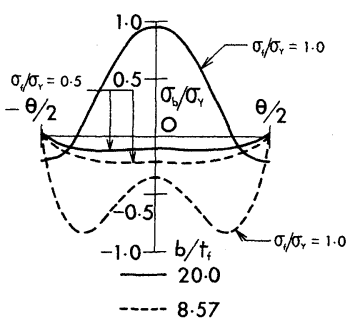

Fig. 14 Nondimensional bending stress $\sigma_{b} / \sigma_{Y}$ along the junction (Type 4).

web deflection mode of a curved I-girder is greatly affected by interaction between the web and the compression flange, particularly by constraint on an upper side of the web. In the present results, torsional buckling of a compression flange causes reduction of constraint on an upper side of a web, so that the difference between aforementioned deflection modes arises in compression region of a web.

Figures. 10 12 show out-of-plane displacements at Point $A$ in compression region in Fig. 2. The results in Figs. 10 and 11 are as to Types 3 and 4. It can be seen from Fig. 10 that after flange buckling occurs, out-of-plane displacement at Point $\mathrm{A}$ increases rapidly. The reason why this phenomenon arises is that constraint on an upper side of a web reduces due to flange buckling. Similar tendency will be observed in Fig. 11. In Fig. 12, a solid line, a long-interval broken line and a doted line indicate curves for Types 1,4 and 5 , among which only curvature parameter $Z$ is different. Comparison among these three curves suggests that curvature affects deflection behavior of a web and that geometrical nonlinearity is pronounced with the increase of curvature.

(3) Distribution of bending stress along an upper side of a web

Distribution of bending stress in circumferencial direction along the upper side of a web is shown in Figs. 13 and 14. In these figures, a solid line indicates results for $b / t_{f}=20.0$ and a broken line for $b / t_{f}=$ 


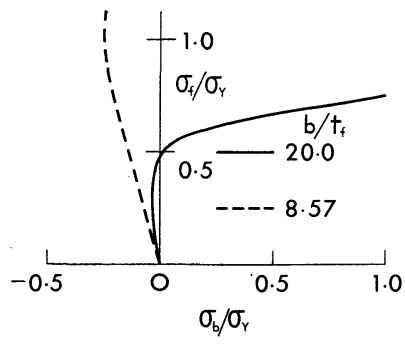

Fig. 15 Relation between $\sigma_{b} / \sigma_{Y}$ and $\sigma_{f} / \sigma_{Y}$ (Type 3).

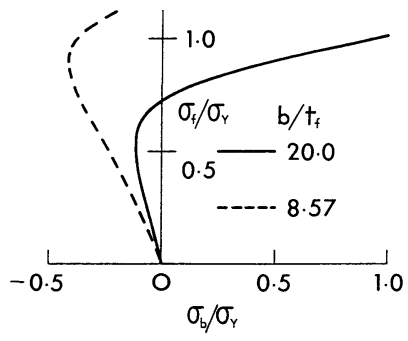

Fig. 16 Relation between $\sigma_{b} / \sigma_{Y}$ and $\sigma_{f} / \sigma_{Y}$ (Type 4).

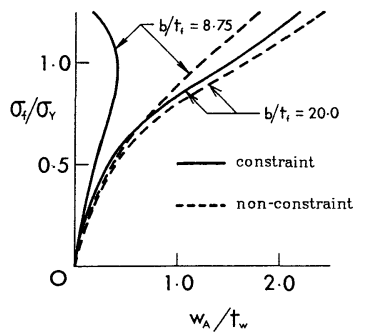

Fig. 17 Difference of web deflection (Point A) between constraint and non-constraint model (Type 4).

8.57, and the sign convention for positive bending stress acting on a web panel indicates the increase of curvature. It is evident from these figures that a sign of bending stress distribution reverses before and after torsional buckling of a flange occurs. This result may be confirmed from Figs. 15 and 16, in which relation between nondimensional flange stress $\sigma_{f} / \sigma_{Y}$ and nondimensional bending stress $\sigma_{b} / \sigma_{Y}$ is plotted. Bending stress at the center point of a girder is considerably large after flange buckling happens. Even though a compression flange does not buckle, concave of bending stress curve near the center point becomes evident as load increases, and the bending stress grows relatively large on the both sides as shown in Fig. 14. The other four types also show similar tendency as the flange stress approaches to yielding stress.

\section{(4) Comments on vertical stiffeners}

In Fig. 17, out-of-plane displacements at Point $\mathrm{A}$ are compared for two models. One is a model that the presence of vertical stiffeners are considered (constraint model) and another is a model that the presence of vertical stiffeners are not considered (non-constraint model). From Fig. 17, it is seen that there is outstanding difference between displacement behaviors of a constraint and a non-constraint model in the case of $b / t_{f}=8.57$, in which flange buckling does not arise. It is expected from this that deflection behavior of a web is influenced by not only a compression flange but also stiffness of vertical stiffeners, while the effects of vertical stiffeners' stiffness on the deflection behavior of a web cannot be evaluated quantitatively, because the finite element representation for vertical stiffeners is not performed in this paper.

\section{CONCLUSIONS}

The following conclusions may be drawn from the present study.

(1) Mixed finite element approach is applicable to the nonlinear analysis of plate assemblies such as curved I-girders without any difficulties, with the help of Lagrange's multipliers method.

(2) The influence of flanges on the behavior of a web cannot be ignored for the determination of strength of curved I-girders. Web deflection behavior depends on constraint by a compression flange on an upper side of a web. Especially, torsional buckling of a compression flange affects markedly deflection behavior of a web.

(3) Aspect ratio of a web panel seems to be of minor importance for the buckling analysis of curved I-girders in the case of curvature parameter $Z \leq 7.15$ and aspect ratio $a / h \geq 1.0$.

(4) Although curvature of a girder is relatively small, that is, curvature parameter $Z$ is such that $Z \leq 7.15$, curvature of a girder affects deflection behavior of a web and geometrical nonlinearity grows noticable as curvature increases.

(5) Torsional buckling of a flange reverses sign of bending stress distribution. Even though flange buckling does not occur, variation of bending stress curve becomes significant with the increase of load.

(6) Sufficient stiffness with respect to a flange is required to avoid torsional buckling of the 
compression flange and to increase overall strength of the curved I-girders. When $Z \leq 7.15$, the requirement of the thickness of outstanding parts of flanges in the Japanese Standard Specifications of Highway Bridges ${ }^{19)}$ may be applied to design a compression flange of curved I-girders, since there is little difference between the flange buckling loads of curved I-girders and those of straight I-girders.

\section{REFERENCES}

1) British Standards Institution : BS 5400, Steel, concrete and composite bridges, Part 3, Code of practice for design of steel bridges, 1982.

2) The Task Committee on Curved Girders of the ASCE-AASHTO Committee on Flexural Members of the Committee on Metals of the Structural Division: Curved I-girder bridge design recommendations, Proc. ASCE, Vol. 103, No. ST5, pp. 1137 1168, May, 1977.

3) Deutscher Ausschuß für Stahlbau : DASt Richtlinie 012, Beulsicherheitsnachweise für Platten, Okt., 1978.

4) Culver, C. G., Dym, C. L. and Brogan, D. K. : Bending behavior of cylindrical web panels, Proc. ASCE, Vol.98, No. ST10, pp. 2291 2308, Oct., 1972.

5) Culver, C. G., Dym, C. L. and Uddin, T. : Web slenderness requirements for curved girders, Proc. ASCE, Vol.99, No. ST3, pp. 417 430, Mar., 1973.

6) Mariani, N., Mozer, J. D., Dym, C. L. and Culver, C. G. : Transverse stiffener requirements for curved webs, Proc. ASCE, Vol.99, No. ST4, pp. 757 771, Apr., 1973.

7) Dabrowski, R. and Wachowiak, J. : Stress in thin cylindrical webs of curved plate girders, Proc. of Colloquium on Design of Plate and Box Girders for Ultimate Strength, London, 1971, IABSE, Vol. 11, pp. 337 352, 1972.

8) Addel-Sayed, G. : Curved webs under combined shear and normal stresses, Proc. ASCE, Vol.99, No. ST3, pp. 511 525, Mar., 1973.

9) Mikami. I., Furuishi, K. and Yonezawa, H. : Nonlinear Behavior of Cylindrical Web Panels under Bending, Proc. of J. S. C. E., No. 299, pp. 23 34, Jul., 1980 (in Japanese).

10) Kuranishi, S. and Hiwatashi. S. : Elastic Behavior of Web Plates of Curved Plate Girders in Bending, Proc. of J. S. C. E., No. 315, pp. 1 11, Nov., 1981 (in Japanese).

11) Nakai, H., Kitada, T. and Ohminami, R. : Experimental Study on Bending Strength of Web Plate of Horizontally Curved Girder Bridges, Proc. of J. S. C. E., No. 340, pp. 19 28, Dec., 1983 (in Japanese).

12) Hiwatashi, S. and Kuranishi, S. : The Finite Displacement Behavior of Horizontally Curved Elastic I-Section Plate Girders under Bending, Proc. of J. S. C. E., No. 350, pp. 59 69, Oct., 1984.

13) Fujii, K. and Ohmura, H. : Nonlinear Behavior of Curved Girder-Web Considered Flange Rigidities, Proc. of J.S. C. E., No. 356, pp. $69 \sim 79$, Apr., 1985.

14) Herrman, L. R. and Campbell, D. M. : A Finite-Element Analysis for Thin Shells, AIAA Journal, Vol. 6, No. 10, pp. 1842 1847, Oct., 1968.

15) Wada, H., Taki, Y., Takamura, T. and Nishimura, T. : Nonlinear Analysis of Plates and Shells by the Incremental Procedure Using Mixed Model of the Finite Element Method. Transactions of J. S. M. E., Apr., 1980 (in Japanese).

16) Nakai, H., Kitada, T., Matsumura, S., Ohminami, R. and Yoshikawa, N. : Inquiries about Web Plates of Curved Girder Bridges, the Bridge and Foundation Engineering, Vol. 15, No. 4, pp. 38 43, May, 1981 (in Japanese).

17) Nakai, H., Kitada, T., Ohminami, R. and Kawai, T. : A Study on Analysis and Design of Web Plate in Curved Girder Bridges subjected to Bending, Proc. of J. S. C.E., No. 368, pp. 235 244, Apr., 1986 (in Japanese).

18) Column Research Committee of Japan : Handbook of Structural Stability, CORONA Publishing Company, LTD., Tokyo, Jun., 1980.

19) Japan Road Association : Standard Specifications for Highway Bridges and Commentary, Part 2 : Steel Bridges, Feb., 1980 (in Japanese).

(Received August 22 1985) 